\title{
Glass patterns as moiré effects: new surprising results
}

\author{
Isaac Amidror \\ Laboratoire de Systèmes Périphériques, Ecole Polytechnique Fédérale de Lausanne, 1015 Lausanne, Switzerland
}

Received August 15, 2002

It is well known that the superposition of two identical random dot patterns may give rise to a particular form of moiré effect known as a Glass pattern. Surprisingly, new research results show that if one chooses appropriate dot shapes for each of the two random dot patterns, while keeping the random dot locations in both layers identical, it is possible to synthesize in the superposition a Glass pattern having any desired shape and intensity profile. (c) 2003 Optical Society of America

OCIS codes: $100.2810,110.2990,120.4120$.

It is widely known that when two identical random dot screens, or any two identical two-dimensional random structures, are superposed on top of each other with a small angle or scaling difference, a typical moiré effect with the shape of a top-viewed funnel appears in the superposition [see Fig. 1(a)]. This moiré effect is known in the literature as a Glass pattern, after Leon Glass, who described it in the late 1960 's. ${ }^{1,2}$ Unlike a moiré effect between periodic dot screens [Fig. 1(b)], which is periodic and extends throughout the entire superposition, ${ }^{3}$ a Glass pattern is concentrated about a certain point in the superposition, and it gradually fades out and disappears as we go farther from this point.

However, in all the cases studied until now, the two superposed random layers were either identical or slightly transformed (scaled, rotated, translated) copies of each other. Here, Glass patterns in the superposition of random dot screens whose dot shapes are not identical are considered. It is shown that by choosing appropriate dot shapes for the dots of the two layers one can synthesize a Glass pattern of any desired shape and intensity profile. This surprising fact is, indeed, an extension of results that are already known for periodic dot screens ${ }^{3}$ into the random case. However, although in the superposition of periodic dot screens the synthesized moiré intensity profiles are periodically repeated throughout the superposition, in the present case the synthesized Glass pattern obtained in the superposition consists of only one moiré intensity profile. Moreover, like any Glass pattern, it is centered about a certain point in the superposition, and it gradually fades out and disappears as we go farther from this point.

Note that the PostScript files that generated the dot screens used in the figures in this Letter are available on the Internet. ${ }^{4}$ They can be downloaded and printed on transparencies with any standard PostScript printer. Superposing these transparencies manually with varying orientations, shifts, etc., can give a vivid demonstration of the Glass (or moiré) patterns and their dynamic behavior in the superposition, even beyond the few static figures in this Letter.

The superposition of two similar two-dimensional aperiodic layers (such as random dot screens) generates a Glass pattern that is concentrated about a certain point in the superposition and gradually disappears farther from this point. Depending on whether it was obtained by rotation of one of the superposed layers, by a scaling transformation, or by a combination of both, it gives rise to an intriguing ordering of the microstructure elements in the superposition in trajectories with a circular, radial, or spiral shape. ${ }^{2}$ However, when we turn one of the superposed aperiodic layers face down on top of the other layer, the Glass pattern disappears.

As was already explained by Glass, this phenomenon occurs because of the local correlation between the structures of the two superposed layers. When two identical layers with the same arbitrary structure are slightly rotated on top of each other [see Fig. 1(a)], a visible Glass pattern is generated about the center of rotation, indicating the high correlation between the two layers in this area: Within the center of the Glass pattern the corresponding elements from each layer fall almost exactly on top of each other, thus generating a brighter area, but slightly away from the center they fall just next to each other, generating a darker area consisting of dot pairs. As we go farther from the center the correlation between the two layers becomes smaller and smaller, and the elements from both layers start falling in an arbitrary, noncorrelated manner; in this area the Glass pattern is no longer visible. This explains why the Glass pattern gradually decays and disappears as we go away from its center. But when

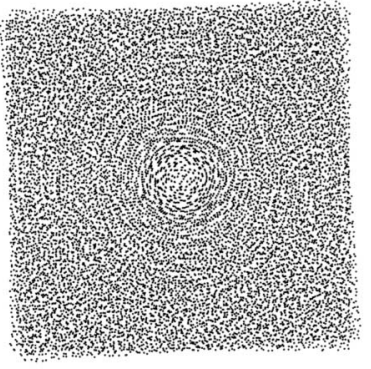

(a)

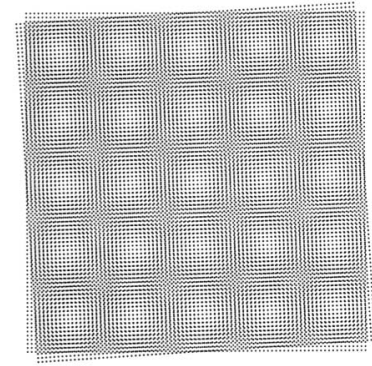

(b)
Fig. 1. (a) Superposition of two identical aperiodic dot screens with a small angle difference gives a moiré effect in the form of a Glass pattern about the center of rotation. (b) When the superposed layers are periodic, a Glass pattern is still generated about the center of rotation, but because of the periodicity of the layers, this pattern is periodically repeated throughout the superposition, thus generating a periodic moiré pattern. 
the two superposed layers are not at all correlated, no Glass pattern appears in the superposition (this is, indeed, what happens when we turn one of the aperiodic layers face down on top of its identical copy).

In all the Glass patterns studied in the past, the two superposed random layers were either identical or slightly transformed (scaled, rotated, or translated) copies of each other. This was required, or at least believed to be required, for guaranteed correlation between the two superposed layers, which is a necessary condition for the generation of a Glass pattern.

However, it is not really required that one have identical or almost identical dot shapes in both random layers to generate a Glass pattern in the superposition; in fact, all that is needed is that the random dot locations be identical (or slightly transformed) in both layers. Thus, if each of the two dot screens consists of dots of a different shape, but the random location of each dot is the same in both layers, the superposition of the two layers will give a clearly visible Glass pattern.

Following previous experience with periodic dot screens (see Ref. 3, Chap. 4), this study is divided into three different cases. Suppose, first, that one of the superposed layers is a random screen consisting of randomly positioned dots with a given desired shape (such as the digit 1) and that the second layer is a random dot screen consisting of tiny pinholes, where the random dot locations in both screens are identical (or slightly transformed). In this case, just as in the superposition of periodic layers (Ref. 3, Sect. 4.4.1), the moiré pattern that appears in the superposition will be a magnified and rotated version of the shape of the individual dots of the first screen. The magnification rate and the orientation of this moiré intensity profile vary according to the angle difference $\alpha$ between the two superposed layers, as in the periodic case. But unlike in the periodic case, the moiré effect generated in the random case is not periodically repeated throughout the superposition, and it consists of only one copy of the magnified dot shape (compare Figs. 2 and 3). In fact, a Glass pattern is obtained that has the shape and the intensity profile of a dot of the first screen (in the present case, the digit 1).

This surprising result seems at first to contradict the basic properties of Glass patterns as they are generally known. As described above, a Glass pattern is expected to be brighter in its center than in areas farther away, because of the partial overlap of the dots of both layers in this area [see Fig. 1(a)]. But the Glass pattern of Fig. 2 seems to contradict this property completely.

In reality, however, there is no contradiction at all. The key point is that in classical Glass patterns, as known until now, the two superposed layers were identical, both consisting of black dots on a white background. But if, as shown in Fig. 2, one of the screens consists of tiny pinholes on a black background, the resulting Glass pattern is the convolution of the dot shape of one layer with the dot shape of the other layer, which, indeed, gives a Glass pattern that has the intensity profile of the dot shape of the other screen (in our case the digit 1). This is similar to the situation in the periodic case (Ref. 3, p. 97), except that in the random counterpart the moiré intensity profile consists of only one copy of the magnified 1-shaped structure.

Now, if we replace our pinhole screen with an inverse-video copy of itself, consisting of tiny black dots on a white (or, rather, transparent) background, the convolution of the individual dot shapes of both layers basically gives an inverse-video version of the result in the first case. Hence, if one of the screens contains tiny black dots, the moiré intensity profile that is obtained is a magnified version of the individual dot shape of the other screen but in inverse video. In our example, a single 1-shaped Glass pattern is obtained that is brighter inside the digit shape and darker outside. Note, however, that this moiré intensity profile is weaker and less impressive than that of the previous case.

Finally, when none of the superposed layers consists of tiny dots (either white or black), the intensity profile form of the resulting moiré (or Glass pattern) is still a magnified version of the convolution of the individual dot shapes of both layers. This convolution gives some kind of blending between the two original dot shapes, but the resulting shape has a blurred or smoothed out appearance resembling a two-dimensional Gaussian, with no recognizable shapes. As we can now understand, this is exactly what happens in classical Glass

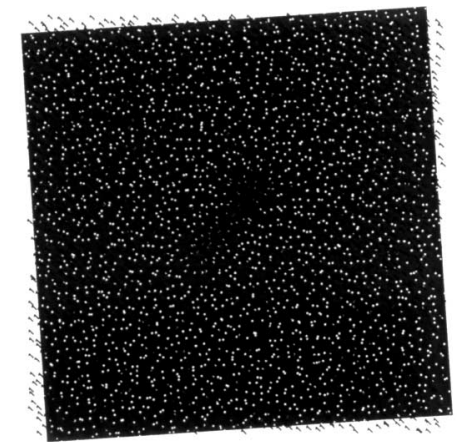

Fig. 2. Superposition of a random dot screen consisting of 1-shaped dots and a random dot screen consisting of small white dots (pinholes), where the dot locations in both screens are identical, gives a single 1-shaped moiré intensity profile (Glass pattern).

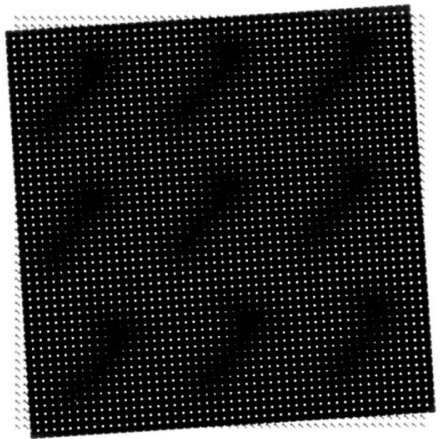

Fig. 3. Periodic counterpart: The superposition of a periodic dot screen consisting of 1-shaped dots and a periodic dot screen consisting of small white dots (pinholes) gives a periodic 1-shaped moiré intensity profile. 
patterns, where the two superposed layers are identical (or where their dot shapes are arbitrary). This is also why the Glass patterns described until now did not have the shape of a magnified version of an element that is randomly repeated in one of the superposed layers.

In conclusion, in spite of their different appearance, moiré effects that occur between periodic or aperiodic dot screens are particular cases of the same basic phenomenon, and all of them satisfy the same fundamental rules. Superpositions of periodic dot screens are, in fact, a particular case in which the dots within each of the layers are arranged periodically. The moiré pattern obtained in this case is simply a periodic repetition of the Glass pattern, as clearly illustrated by Figs. 2 and 3 .

Moreover, wehave shown that classical Glass patterns, as widely known from literature, are simply a particular case that occurs when the dot shapes in both random layers are arbitrary. But by choosing appropriate dot shapes for the dots of the two layers we can synthesize a Glass pattern of any desired shape and intensity profile.
These surprising results can find interesting applications in several scientific and technological disciplines. For example, they can be used in vision research, in physiological experiments such as form perception, and in the study of the human visual system, where classical Glass patterns have already been used for a long time., ${ }^{2,5}$ Possible technological fields of application may include precision optical alignment, image registration, measurement of microscopic displacements, ${ }^{6}$ analogic magnifiers, and even document security and authentication. ${ }^{7}$

\section{References}

1. L. Glass, Nature 223, 578 (1969).

2. L. Glass and R. Pérez, Nature 246, 360 (1973).

3. I. Amidror, The Theory of the Moiré Phenomenon (Kluwer, Dordrecht, The Netherlands, 2000).

4. http://spwww.epfl.ch/books/moire/kit.html.

5. S. C. Dakin, Vision Res. 37, 2227 (1997).

6. K. Patorski, Handbook of the Moiré Fringe Technique (Elsevier, Amsterdam, 1993), pp. 99-139.

7. I. Amidror, Proc. SPIE 4677, 89 (2002). 